PROCEEDINGS OF THE

AMERICAN MATHEMATICAL SOCIETY

Volume 126, Number 9, September 1998, Pages 2687-2690

S 0002-9939(98)04730-3

\title{
A COUNTEREXAMPLE TO THE BARTLE-GRAVES SELECTION THEOREM FOR MULTILINEAR MAPS
}

\author{
CECÍLIA S. FERNANDEZ
}

(Communicated by Theodore W. Gamelin)

\begin{abstract}
We present an example showing that the multilinear version of the Bartle-Graves Selection Theorem is false, even on finite dimensional spaces.
\end{abstract}

\section{INTRODUCTION}

The Bartle-Graves Selection Theorem [1] in the context of Banach spaces can be formulated as follows (see also [2] or [3]):

If $E$ and $F$ are Banach spaces and $u: E \rightarrow F$ is continuous, linear and surjective, then there exists a continuous function $g: F \rightarrow E$ such that $u \circ g$ is the identity map on $F$.

It is natural to ask whether the multilinear version of the above result remains true. The goal of the present work is to show that this is not the case. More precisely, we shall present a continuous bilinear onto map which does not have a continuous right inverse.

\section{MAin RESUlt}

Theorem. There are Banach spaces $E_{1}, E_{2}, F$ and a continuous bilinear map from $E_{1} \times E_{2}$ onto $F$ which does not have a continuous right inverse.

Proof. Let $\mathcal{P}_{j}(\mathbf{C})$ denote the set of all complex polynomials with degree $\leq j$, normed by

$$
\|p\|=\max _{0 \leq k \leq j}\left|a_{k}\right| \quad\left(p(z)=a_{0}+a_{1} z+\ldots+a_{j} z^{j} \in \mathcal{P}_{j}(\mathbf{C})\right) .
$$

Clearly, $\mathcal{P}_{j}(\mathbf{C})$ is a Banach space. Consider $\mathcal{P}_{j}(\mathbf{C}) \times \mathcal{P}_{j}(\mathbf{C})$ normed by

$$
\|(p, q)\|=\max \{\|p\|,\|q\|\} \quad\left(p, q \in \mathcal{P}_{j}(\mathbf{C})\right)
$$

and let

$$
u:(p, q) \in \mathcal{P}_{1}(\mathbf{C}) \times \mathcal{P}_{1}(\mathbf{C}) \mapsto p q \in \mathcal{P}_{2}(\mathbf{C}),
$$

where $p q$ denotes the usual multiplication between the polynomials $p$ and $q$. Clearly, $u$ is a continuous bilinear map. Moreover, the Fundamental Theorem of Algebra allows us to conclude that $u$ is onto.

Received by the editors January 28, 1997.

1991 Mathematics Subject Classification. Primary 46B99.

Key words and phrases. Continuous selections, multilinear mappings.

(C) 1998 American Mathematical Society 
Let $g$ be a right inverse of $u$. Then, for $a, b, c \in \mathbf{C}, a \neq 0$, we must have

$$
g\left(a z^{2}+b z+c\right)=\left(\alpha(a, b, c)\left(z-\frac{-b-h(a, b, c)}{2 a}\right), \beta(a, b, c)\left(z-\frac{-b+h(a, b, c)}{2 a}\right)\right),
$$

where $h(a, b, c)$ is a square root of $b^{2}-4 a c$, and $\alpha(a, b, c)$ and $\beta(a, b, c)$ are complex numbers such that $\alpha(a, b, c) \beta(a, b, c)=a$.

Suppose that $g$ is continuous and consider the following function:

$$
\varphi: c \in \mathbf{C} \mapsto \frac{h(1,0,-c)}{2} \in \mathbf{C} .
$$

Clearly, $[\varphi(c)]^{2}=c$ for all $c \in \mathbf{C}$. Moreover, $\varphi$ is continuous. Indeed, let $c_{0} \in \mathbf{C}$ and put $\alpha_{0}=\alpha\left(1,0,-c_{0}\right)$. Let $0<\epsilon<\frac{\left|\alpha_{0}\right|}{2}$ and $\epsilon_{1}=\min \left\{\epsilon, \frac{\left|\alpha_{0}\right| \epsilon}{2\left(1+\left|\varphi\left(c_{0}\right)\right|\right)}\right\}$. Since $g$ is continuous at the polynomial $z^{2}-c_{0}$, there is a $\delta>0$ such that

$$
\left\|g\left(z^{2}-c\right)-g\left(z^{2}-c_{0}\right)\right\|<\epsilon_{1} \quad \text { whenever }\left|c-c_{0}\right|<\delta .
$$

By (1), we have that

$$
g\left(z^{2}-c_{0}\right)=\left(\alpha_{0}\left(z+\varphi\left(c_{0}\right)\right), \frac{1}{\alpha_{0}}\left(z-\varphi\left(c_{0}\right)\right)\right)
$$

and

$$
g\left(z^{2}-c\right)=\left(\alpha(z+\varphi(c)), \frac{1}{\alpha}(z-\varphi(c))\right),
$$

where $\alpha=\alpha(1,0,-c)$. So, by (2) and the definition of the norm on $\mathcal{P}_{1}(\mathbf{C})$, we get that

$$
\left|\alpha_{0} \varphi\left(c_{0}\right)-\alpha \varphi(c)\right|<\epsilon_{1} \text { and }\left|\alpha-\alpha_{0}\right|<\epsilon_{1} \quad \text { whenever }\left|c-c_{0}\right|<\delta .
$$

By the above inequalities and the choice of $\epsilon_{1}$ we finally get that $\left|\varphi(c)-\varphi\left(c_{0}\right)\right|<\epsilon$ whenever $\left|c-c_{0}\right|<\delta$. This proves the continuity of $\varphi$ at $c_{0}$. Since $c_{0}$ is arbitrary in $\mathbf{C}, \varphi$ is continuous.

Thus, $\varphi$ is a continuous branch of the square root on the whole complex plane. But this cannot happen (cf. [4], page 183). Therefore, $g$ is not continuous. Since $g$ is an arbitrary right inverse for $u$, we have the desired result.

\section{SOME REMARKS}

3.1. By identifying $\mathcal{P}_{j}(\mathbf{C})$ canonically with $\mathbf{C}^{j+1}$, we can regard the mapping $u$ defined in the proof of the Theorem as follows:

$$
u:((a, b),(c, d)) \in \mathbf{C}^{2} \times \mathbf{C}^{2} \mapsto(a c, a d+b c, b d) \in \mathbf{C}^{3} .
$$

Since $\mathbf{C}^{n}$ is canonically isomorphic to $\mathbf{R}^{2 n}$, we can then see $u$ as a continuous bilinear map from $\mathbf{R}^{4} \times \mathbf{R}^{4}$ onto $\mathbf{R}^{6}$, which does not have a continuous right inverse (by the Theorem). Thus, the Bartle-Graves Selection Theorem also fails for bilinear maps in the real case. 
3.2. For all $m \geq 2$, there are Banach spaces $E_{1}, \ldots, E_{m}, F$ and a continuous $m$ linear map from $E_{1} \times \ldots \times E_{m}$ onto $F$ which does not have a continuous right inverse. Indeed, the case $m=2$ is the Theorem. Now, let $m \geq 3$. Consider

$$
v:\left(\lambda_{1}, \ldots, \lambda_{m-2}, p, q\right) \in \underbrace{\mathbf{C} \times \ldots \times \mathbf{C}}_{m-2} \times \mathcal{P}_{1}(\mathbf{C}) \times \mathcal{P}_{1}(\mathbf{C}) \mapsto \lambda_{1} \cdots \lambda_{m-2} p q \in \mathcal{P}_{2}(\mathbf{C}) .
$$

Clearly, $v$ is a continuous $m$-linear surjective map. Now, let $f$ be a right inverse for $v$. Then, for $a, b, c \in \mathbf{C}, a \neq 0$, we have that

$$
\begin{aligned}
f\left(a z^{2}+b z+c\right)= & \left(\lambda_{1}(a, b, c), \ldots, \lambda_{m-2}(a, b, c),\right. \\
& \left.\alpha(a, b, c)\left(z-\frac{-b-h(a, b, c)}{2 a}\right), \beta(a, b, c)\left(z-\frac{-b+h(a, b, c)}{2 a}\right)\right),
\end{aligned}
$$

where $h(a, b, c)$ is a square root of $b^{2}-4 a c$ and $\alpha(a, b, c)$ and $\beta(a, b, c)$ are complex numbers satisfying $\lambda_{1}(a, b, c) \cdots \lambda_{m-2}(a, b, c) \alpha(a, b, c) \beta(a, b, c)=a$. If $f$ were continuous, the argument used in the proof of the Theorem would imply that the function $c \in \mathbf{C} \mapsto \frac{h(1,0,-c)}{2} \in \mathbf{C}$ is a continuous branch of the square root on $\mathbf{C}$, which would be a contradiction. Thus, the Bartle-Graves Selection Theorem fails for $m$-linear maps whenever $m \geq 2$.

3.3. Let $f$ be a continuous linear surjective map. The fact that $f$ has a continuous right inverse is equivalent to the fact that $f$ is open at the origin. We shall verify that this equivalence can fail if $f$ is an $m$-linear map with $m \geq 2$. For this, consider the following:

Proposition. The mapping $v$ defined in remark 3.2 is open at the origin.

Proof. It is clearly enough to show that the mapping $u$ defined in the proof of the Theorem is open at the origin. For this purpose, fix $0<\epsilon<1$. Let $\delta>0$ be such that $\delta<\epsilon^{4} / 2$ and

$$
\left|-B \pm \sqrt{B^{2}-4 A C}\right|<\epsilon^{2} \quad \text { whenever }|A|<\delta,|B|<\delta \text { and }|C|<\delta .
$$

Fix $A, B, C \in \mathbf{C}$ with $|A|<\delta,|B|<\delta$ and $|C|<\delta$. The proposition will follow as soon as we establish the existence of points $a, b, c, d \in \mathbf{C}$ with

$$
|a|<\epsilon,|b|<\epsilon,|c|<\epsilon,|d|<\epsilon \text { and }(a z+b)(c z+d)=A z^{2}+B z+C .
$$

If $A=0$, it is enough to put $a=0, b=\epsilon^{2} / 2, c=B / b$ and $d=C / b$. Thus, assume $A \neq 0$. In order to have $(a z+b)(c z+d)=A z^{2}+B z+C$, it must be the case that

$$
a z+b=\alpha\left(z-r_{1}\right) \text { and } c z+d=\beta\left(z-r_{2}\right),
$$

where $\alpha \beta=A$ and $r_{1}, r_{2}$ are the roots of $A z^{2}+B z+C$ (which implies $a=\alpha$, $b=-\alpha r_{1}, c=\beta$ and $d=-\beta r_{2}$ ). Without loss of generality, we may assume

$$
r_{1}=\frac{-B+\sqrt{B^{2}-4 A C}}{2 A} \text { and } r_{2}=\frac{-B-\sqrt{B^{2}-4 A C}}{2 A} .
$$

So, our problem is reduced to finding $\alpha, \beta \in \mathbf{C}$ such that $\alpha \beta=A,|\alpha|<\epsilon,|\beta|<\epsilon$, $\left|\alpha r_{1}\right|<\epsilon$ and $\left|\beta r_{2}\right|<\epsilon$. We have two cases:

Case 1. $\left|r_{1}\right|<\epsilon$ or $\left|r_{2}\right|<\epsilon$ :

If $\left|r_{1}\right|<\epsilon$, then put $\alpha=\frac{\epsilon}{2}$ and $\beta=\frac{2 A}{\epsilon}$; else put $\alpha=\frac{2 A}{\epsilon}$ and $\beta=\frac{\epsilon}{2}$.

Case 2. $\left|r_{1}\right| \geq \epsilon$ and $\left|r_{2}\right| \geq \epsilon$ :

In this case, $\alpha=\frac{\sqrt{C}}{r_{1}}$ and $\beta=\frac{\sqrt{C}}{r_{2}}$ will do the trick. 


\section{ACKNOWLEDGMENT}

The present work is part of the author's Ph.D. Thesis at Kent State University. The subject of this work was suggested by Professor Richard M. Aron, to whom the author is deeply grateful. The author would like also to thank CAPES/Brazil for financial support.

\section{REFERENCES}

[1] R. G. Bartle and L. M. Graves, Mappings Between Function Spaces, Trans. Amer. Math. Soc. 72 (1952), 400-413. MR 13:951i

[2] C. Bessaga and A. Pelczynski, Selected Topics in Infinite Dimensional Topology, Monografie Matematyczne 58, Polish Scientific Publishers, Warszawa (1975). MR 57:17657

[3] N. Bourbaki, Topological Vector Spaces, Springer-Verlag (1987). MR 88g:46002

[4] B. P. Palka, An Introduction to Complex Function Theory, Springer-Verlag (1991). MR 92b:30001

Departamento de Matemática, Universidade Federal do Espírito Santo, Av. FerNANDO Ferrari S/N. 29060-900, Vitória, ES, Brasil

Current address: Department of Mathematics and Computer Science, Kent State University, Kent, Ohio 44242 